\title{
Uso de tuberculina en caninos para buscar reservorios de Mycobacterium bovis
}

\section{Tuberculin use in canines to search for Mycobacterium bovis reservoirs}

Fecha de Recepción: 10 de octubre de 2012

Fecha de Aceptación: 18 de septiembre de 2013

Carlos Bernal Suarez', Sebastián Martínez Garcia',

Roy José Andrade-Becerra ${ }^{2}$

\section{Resumen}

Objetivo. Utilizar la prueba de tuberculina en caninos para buscar reservorios de Mycobacterium bovis. Materiales y métodos. Se utilizó la prueba comparada en los caninos que dieron positivo a la PPD ano-caudal, y la técnica de Ziehl-Neelsen en los animales dudosos, para corroborar el resultado. Resultados. Los caninos no son reservorios de la enfermedad, por tal razón, se sugiere utilizar pruebas diagnósticas más específicas para identificar el Mycobacterium en ellos.

Palabras clave: Mycobacterium bovis, Hospedero, PPD bovina, Ziehl-Neelsen (Fuente: DeCS).

\begin{abstract}
Objective. The proposal to prevent tuberculosis is to use the tuberculin in canines, to search for Mycobacterium bovis reservoirs. Materials and Methods. The compared test was used for those canines that were positive to PPD anus-flow, and Ziehl-Neelsen technique in doubtful animals to confirm the test. Results. The canines are not the disease reservoirs, for that reason we suggested to use more specific diagnostic tests for the Mycobacterium in canines identification.
\end{abstract}

Key words: Mycobacterium bovis, Host, Bovine PPD, Ziehl-Neelsen (Source: DeCS).

1 Médico Veterinario Zootecnista, Profesional Independiente. carlosbernal73@hotmail.com; sebastianmaga@hotmail.com

2 Médico Veterinario, MCs., Ph.D. Docente Universidad Pedagógica y Tecnológica de Colombia, Tunja.

royjandrade@yahoo.es 


\section{Introducción}

La tuberculosis bovina es una enfermedad bacteriana crónica, de animales y del hombre, causada por Mycobacterium bovis, y se caracteriza, normalmente, por la formación de granulomas nodulares, conocidos como tubérculos. En muchos países, la tuberculosis bovina es una enfermedad infecciosa importante en el ganado vacuno, que es el hospedero de mantenimiento de las bacterias; pero las infecciones también se han descrito en otros animales, como borregos, cabras, caballos, cerdos, venados, perros y gatos (1). El Mycobacterium bovis es un microorganismo zoonótico, y debe ser tratado como un microorganismo del grupo III de riesgo/peligro, para evitar que se produzca infección en humanos.

La región norte del departamento de Cundinamarca y la occidental de Boyacá, por lo menos una parte , por ser especializadas en ganadería de leche, son afectadas por la tuberculosis bovina. EI ICA, en el 2009, ordenó el sacrificio de 120 animales de los municipios de San Miguel de Sema, Chiquinquirá, Simijaca y Susa; de igual manera, durante 2006 fueron sacrificados 220 animales en la zona de Fúquene; pese a esto, ninguno de los dos departamentos (Cundinamarca y Boyacá), ni el país, ha sido declarado libre de tuberculosis, lo que causa un detrimento económico al país (2).

Estudios sobre la prevalencia de la tuberculosis en la vereda Pantano, del municipio de Simijaca (Cundinamarca), indicaron que el $1.57 \%$ de la población analizada en el año 2007 resultó positiva a Mycobacterium bovis (3); así mismo, en trabajos realizados en Chiquinquirá y Saboyá (Boyacá) se obtuvo una prevalencia del 1.53\% (4). Los programas gubernamentales para controlar y erradicar la Tuberculosis Bovina utilizan un esquema de aplicación intradérmica de la tuberculina (PPD) y el sacrificio de los animales reactores; sin embargo, se estima que hasta el $19 \%$ de ganado tuberculoso no reacciona a la tuberculina (5); por esto, se empleó la PPD Bovina en los caninos, en búsqueda de reservorios de Mycobacterium, observando el tipo de reacción que presentaban.

\section{Materiales y métodos}

Tipo de estudio. Se realizó un estudio probabilístico con muestreo aleatorio simple. Para la recolección de datos se utilizó un diario de campo en el que se describieron de manera detallada las actividades y resultados; posteriormente, la información recolectada fue sistematizada en una hoja de cálculo de Microsoft Office Excel Versión 2007, y a los datos obtenidos se les realizó la prueba de chi $^{2}$ (chi cuadrado) de bondad de ajuste con el paquete estadístico SPSS 17.

Metodología. En la primera fase se empleó la prueba de sensibilidad retardada PPD Bovina en el pliegue ano-caudal de los caninos, conforme con las directrices establecidas por el Instituto Colombiano Agropecuario (ICA); en los casos obtenidos como sospechosos y positivos a la PPD Bovina se utilizó la PPD Bovina como prueba cervical simple comparativa (segunda fase); para los casos positivos y dudosos, tanto en PPD Bovina ano-caudal como en PPD Bovina cervical, se realizó toma de esputo y se empleó la técnica de Ziehl-Neelsen para corroborar la presencia de Bacilos Ácido Alcohol Resistentes (BAAR) (tercera fase).

\section{Resultados y discusión}

\section{Primera fase}

Se realizó la prueba de chi $^{2}$ (chi cuadrado) de bondad de ajuste para determinar si había diferencia significativa entre las frecuencias de individuos con respuesta negativo (67 individuos), dudoso (11 individuos) y positivo (1 individuo) en la aplicación de PPD en el pliegue caudal. La prueba indicó que había por lo menos una frecuencia diferente $(p=1.35 \mathrm{E}-21 ; p<0.05)$; se determinaron los porcentajes para cada grupo: negativos, $84.8 \%$; dudosos, $13,9 \%$, y positivos, 1,3\% (Tabla 1).

Lo anterior coincide con el estudio realizado por Vergara (2004), en el que se encontraron dos caprinos con reacción positiva a la PPD Bovina, prueba ano-caudal, y se determinó una prevalencia del $0,67 \%$, indicando la presencia de 
Mycobacterium bovis. Asumiendo estos resultados, se aplicó la PPD Bovina prueba cervical simple en los casos dudosos y positivos, para corroborar la presencia de Mycobacterium bovis, teniendo en cuenta lo establecido por Bernardelli (2007), quien refiere que la prueba presenta reacción cruzada con otros Mycobacterium.

TABLA 1. REACCIÓN DE LA TUBERCULINA PPD BOVINA EN CANINOS, PRUEBA PLIEGUE ANOCAUDAL

\begin{tabular}{|c|c|c|}
\hline Reacción & $\begin{array}{c}\text { Porcentaje } \\
\text { (\%) }\end{array}$ & $\begin{array}{c}\text { Número } \\
\text { de } \\
\text { animales }\end{array}$ \\
\hline Negativa & 84.81 & 67 \\
\hline $\begin{array}{c}\text { No superior a 2 mm } \\
\text { Dudosa }\end{array}$ & 13.92 & 11 \\
\hline $\begin{array}{c}\text { omprendido entre } \\
2 \text { y } 4 \text { mm }\end{array}$ & 1.26 & 1 \\
\hline Positiva & \\
\hline
\end{tabular}

Mediante la prueba de Fisher se determinó que las proporciones eran estadísticamente iguales, es decir, los casos dudosos y positivos se podían presentar en machos o hembras $(p=0.5995$; $p \geq 0.05$ y $p=0.2206 ; p \geq 0.05$, respectivamente).

\section{Segunda fase}

Los resultados obtenidos permitieron observar que el 54,5\% presentó una reacción negativa a la prueba cervical con PPD bovino; el 45,5\% mostró una reacción dudosa, y ninguno de los caninos presentó una reacción positiva. Resultados similares se encontraron en la investigación de Fernández y Rosadio (1998), la cual realizó una tuberculinización en 651 alpacas, con resultados negativos, y tampoco obtuvo respuesta de hipersensibilidad, empleando la región axilar, equivalente en los camélidos a la región cervical utilizada por este estudio en los caninos, corroborando la similitud de la investigación anterior.

La aplicación de la prueba cervical en los animales con reacción positiva a la prueba anocaudal mostró una reacción negativa (Figura 1) debido, probablemente, a una reacción cruzada con otro Mycobacterium, tal como Bernardelli (2007) lo reportó. Por lo tanto, aun cuando la prueba tiene una buena sensibilidad, en trabajo de campo puede mostrar reacción cruzada para cualquier Mycobacterium sin importar la especie (9); consecuentemente, se puede tratar de respuestas cruzadas debido a Mycobacterias escotocromógenas o Mycobacterias no tuberculosas.

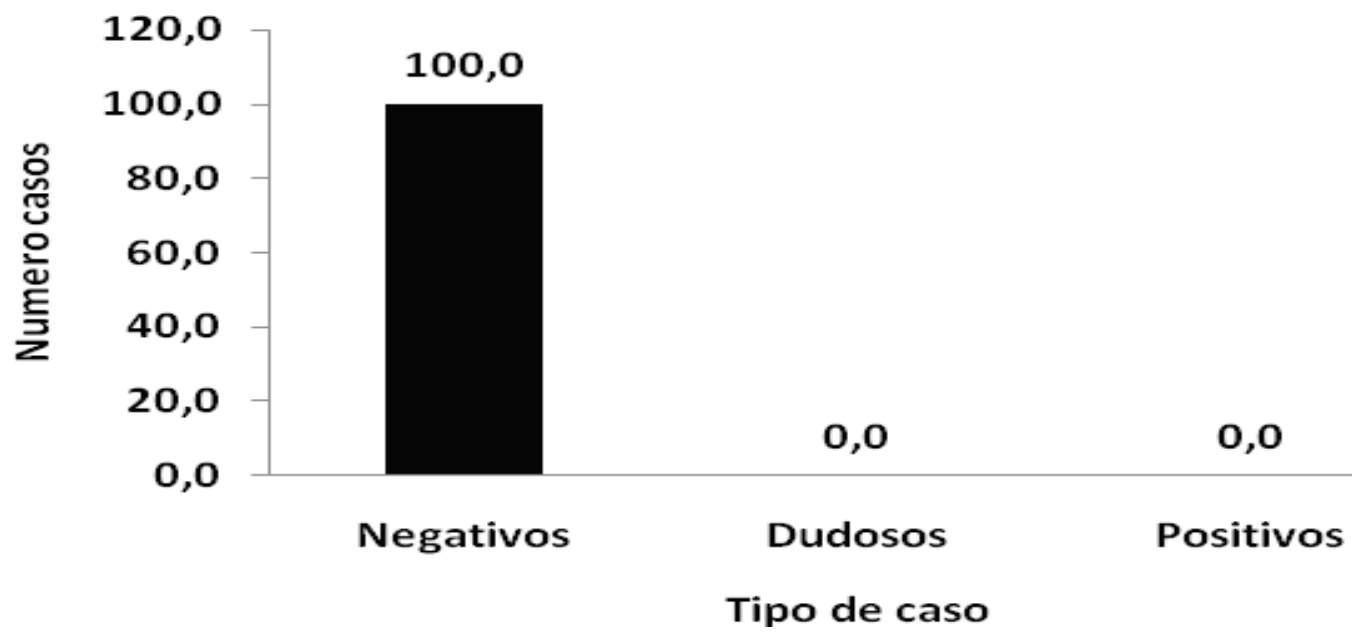

FIGURA 1. REACCIÓN DE LA TUBERCULINA PPD BOVINA EN CANINOS, PRUEBA CERVICAL AL GRUPO DE POSITIVOS 


\section{Tercera fase}

Se decidió implementar la técnica de Ziehl-Neelsen a los 12 caninos que presentaron una reacción de hipersensibilidad, tanto dudosa como positiva, a la prueba ano-caudal y cervical simple. Los esputos se llevaron al laboratorio, se hicieron extendidos sobre láminas portaobjetos y se aplicaron los reactivos de la prueba de Ziehl-Neelsen, dando como resultado la negatividad de las 12 pruebas con conteos inferiores a 1 BAAR/300 campos; el procedimiento fue realizado 2 veces, tal y como lo sugiere Canese (2009), demostrando que no existe presencia de BAAR en la población canina objeto de este estudio.

\section{Referencias bibliográficas}

(1) Organización Mundial de Sanidad Animal OIE. Manual de la OIE sobre animales terrestres. Tuberculosis Bovina (en línea) 2004 (fecha de acceso: 28 de agosto de 2009). URL disponible en http://www.oie.int/esp/normes/mmanual/ pdf_es/2.3.03Tuberculosis_bovina.pdf

(2) Ministerio de Agricultura y Desarrollo Rural. Informe hatos libres de tuberculosis. Balance Cundinamarca y Boyacá; 2009.

(3) Pava-Fernández DY. Determinación de la prevalencia de Tuberculosis Bovina en hatos lecheros en la vereda Pantano del municipio de Simijaca (Cundinamarca). Facultad de Ciencias Agropecuarias, Universidad Pedagógica y Tecnológica de Colombia, 2008.
(4) Ospina N, Martínez M. Prevalencia de tuberculosis bovina en hembras mayores de 24 meses y machos mayores de 38 meses en la cuenca lechera de Chiquinquirá y Saboyá. Facultad de Ciencias Agropecuarias, Universidad Pedagógica y Tecnológica de Colombia, 2007.

(5) Estrada-Chávez C Mancilla R. Determinación de anticuerpos anti-PPD en hatos lecheros con distintas prevalencias de tuberculosis bovina en México. Vet Mex 2010; 32(3): 207-211.

(6) Vergara-Manrique GE. Prevalencia de la tuberculosis caprina en la provincia de barranca. Vet Mex 2009; 31(2): 117-124.

(7) Bernardelli A. Producción y control de tuberculina bovina y aviar. Derivado Proteico Purificado (DPP) (en línea) 2007 (fecha de acceso: 20 de octubre de 2011). URL disponible en: www.senasa.gov.ar/Archivos/ File/File1011-tuberbov.pdf

(8) Fernández M, Rosadio R. Aplicación de Tuberculinas en Alpacas. Rev Inv Pec 2008; 9(2): 138-143.

(9) Instituto Colombiano Agropecuario ICA. Informe técnico de sanidad (en línea) 2008 (fecha de acceso: 25 de octubre de 2011). URL disponible en: www.ica.gov.co

(10) Canese A. Manual de microbiología y parasitología médica. 5 Edición. Editorial Panamericana. Paraguay; 2009. 